\title{
Brain abscess as a manifestation of spinal dermal sinus
}

\author{
Parisa Emami-Naeini \\ Ali Mahdavi \\ Hamed Ahmadi \\ Nima Baradaran \\ Farideh Nejat
}

Department of Neurosurgery, Children's Hospital Medical Center, Medical Sciences/University of Tehran, Tehran, Iran
Correspondence: Farideh Nejat

Tehran, F. Nejat, I 4155-7854

Tel +98 9l 21494064

Fax+98216930024

Email nejat@sina.tums.ac.ir

\begin{abstract}
Dermal sinuses have been associated with a wide spectrum of clinical manifestations ranging from asymptomatic to drainage of purulent material from the sinus tract, inclusion tumors, meningitis, and spinal abscess. To date, there has been no documented report of brain abscess as a complication of spinal dermal sinus. Here, we report an 8-month-old girl who was presented initially with a brain abscess at early infancy but lumbar dermal sinus and associated spinal abscess were discovered afterwards. The probable mechanisms of this rare association have been discussed.
\end{abstract}

Keywords: brain abscess, spinal dermal sinus, spinal abscess

\section{Introduction}

Congenital spinal dermal sinus is an innocuous-appearing form of spinal dysraphism that can nevertheless produce significant morbidity if not managed in an appropriate fashion. The skin manifestation of a dermal sinus usually consists of a hairline ostium in the midline neuraxis. This skin defect represents the superficial stoma of an epithelium-lined tract that may extend into the intraspinal neural elements and provides an avenue for ingress of bacteria, leading to recurrent bacterial meningitis (Matson and Jerva 1966; Keating et al 2004).

Brain abscess, defined as a focal suppurative infection within the brain parenchyma, is relatively uncommon with an incidence of about 1 in 100,000 persons per year (Roos and Tyler 2005). Different conditions may render the patient susceptible to brain abscess including infection of adjacent organs, meningitis, cranial dermal sinuses, and congenital heart disease. Spinal abscess is also an uncommon infection of the nervous system. It may result from hematogenous bacterial dissemination or local extension of an infected adjacent dermal sinus tract (Morandi et al 1999; Bunyaratavej et al 2006).

Lumbar dermal sinuses may give rise to spinal abscesses (Bean et al 1979; Bunyaratavej et al 2006), but there is no documented report of brain abscess as a complication of spinal dermal sinus. Here, we report an 8-month-old girl who presented initially with a brain abscess in early infancy but lumbar dermal sinus and associated spinal abscess were discovered three months later.

\section{Case report}

A previously healthy 8-month-old girl was admitted with a 2-week history of fever, irritability, and vomiting together with progressive right hemiparesis. Brain computed tomography (CT) scan revealed extensive left frontoparietal hypodensity along with unilateral exvacuum ventriculomegaly, containing multiple separated cysts with variable density, without midline shift (Figure1). Brain magnetic resonance imaging (MRI) demonstrated the same lesions with bright ring enhancement (Figure 2). Due to her critical general condition, multiple abscesses were drained through a burr hole. 


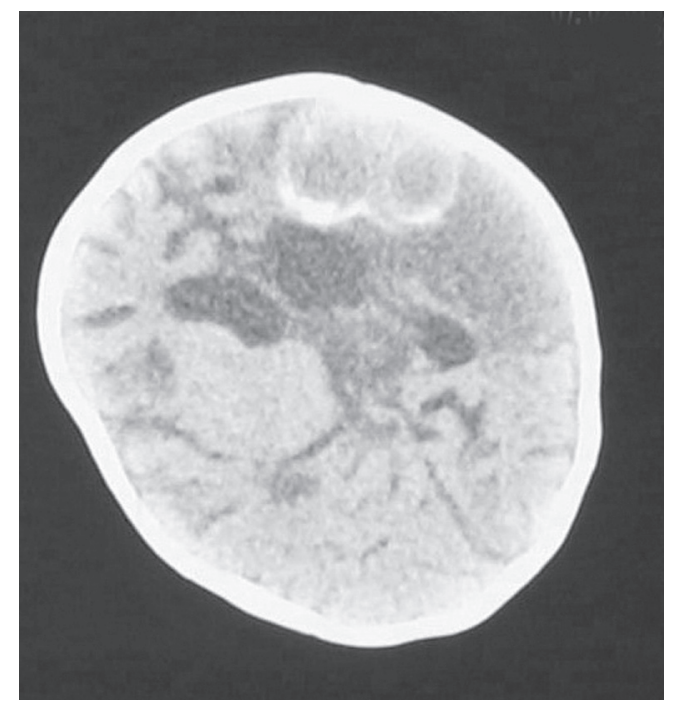

Figure I The first brain computed tomography scan without contrast shows multiple brain abscesses with variable density and surrounding extensive hypodensity.

The specimen contained many polymorphonuclear cells and the culture was positive for Escherichia coli. Antibiotic therapy with ceftriaxone, vancomycin and metronidazole in meningeal dose was initiated and continued for eight weeks. She was followed with repeated brain CT scans which showed progressive shrinkage of all cavities along with profound brain malacia around the previous abscesses (Figure 3). Extensive evaluation for the source of infection (contiguous, distant site, and systemic) were negative. Good recovery of all neurological deficits were achieved and she was followed every two weeks in outpatient setting. Four weeks later she became febrile and irritable with severe back discomfort

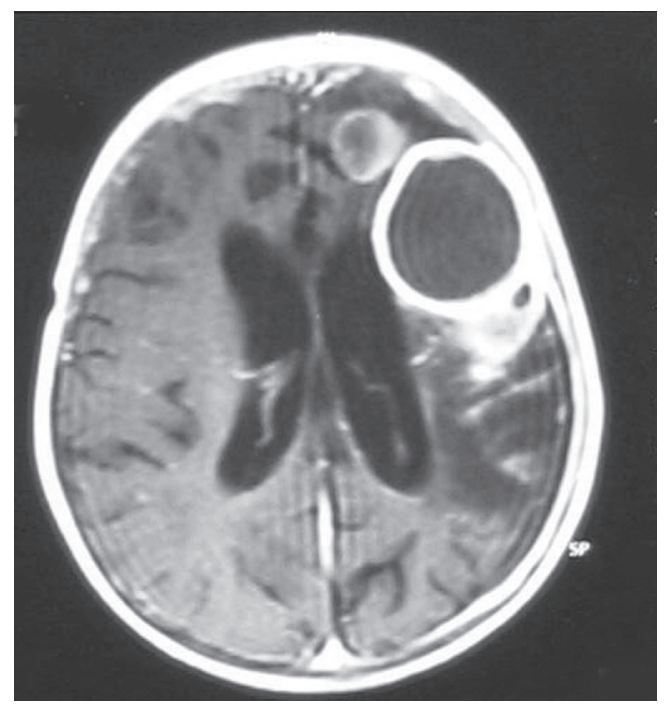

Figure 2 Brain magnetic resonance image (axial view, TI-weighted image with gadolinium) reveals multiple abscesses with regular ring enhancement. without any history of trauma. She was admitted immediately and examination revealed severe back tenderness without swelling or redness but opposed to our expectations, there was a midline pinpoint dermal sinus in lumbosacral area with purulent discharge (which had neither been apparent on the first admission nor visible to her mother). The rest of neurological examination appeared normal. With the primary impression of spinal abscess, ceftriaxon $(100 \mathrm{mg} / \mathrm{kg})$ and amikacin $(15 \mathrm{mg} / \mathrm{kg})$ were prescribed. Laboratory test revealed erythrocyte sedimentation rate of 85 , leukocyte count of 16,000 with $90 \%$ neutrophil and negative blood cultures. Spinal MRI was requested but owing to development of progressive paraparesis and urinary retention within two days, she underwent urgent decompressive surgery.

The pin point sinus had a tract that crossed L5 lamina rostrally and traversed the dura mater, ended intradurally in a dermoid tumor containing keratin, thin hair, and pus. Osteoplastic laminotomy was begun from L5 and extended to T12 in order to achieve complete resection of tumor and associated abscess. There was severe adhesion between pia mater and dura mater. All related roots were inflamed and displaced ventrolaterally by the mass of purulent dermoid tumor. At the level of conus (L1) the cord was very tense and abscess was ingressed intramedullary which was decompressed. The culture taken from the tumor specimen was positive for $E$. coli. Antibiotic therapy was continued for another six weeks. Spinal MRI two days after operation revealed severe arachnoiditis without intradural mass (Figure 4). She recovered completely within two weeks of the operation. Two years after treatment, she is free of disease, with normal neurological examination and imaging.

\section{Discussion}

Dermal sinuses have been associated with a wide spectrum of clinical manifestations ranging from asymptomatic to serious complications. About $75 \%$ of dermal sinuses present with symptoms attributable to the involvement of the central nervous system. Some patients may present with complaints of drainage of purulent material from the sinus tract. Inclusion tumors associated with dermal sinuses are another manifestation. Nineteen per cent of patients display evidence of both tumor and infection (Keating et al 2004; Ansari et al 2006). Meningitis and spinal abscess are not uncommon (Matson and Jerva 1966; Bean et al 1979; Bunyaratavej et al 2006).

The appearance of the ostium of a dermal sinus is variable, and the stoma may be obscured by a hairy patch or skin nevus. In $6 \%$ to $7 \%$ of cases, the tract reportedly terminates dorsal to the spinal elements; in $10 \%$ to $20 \%$, it terminates 


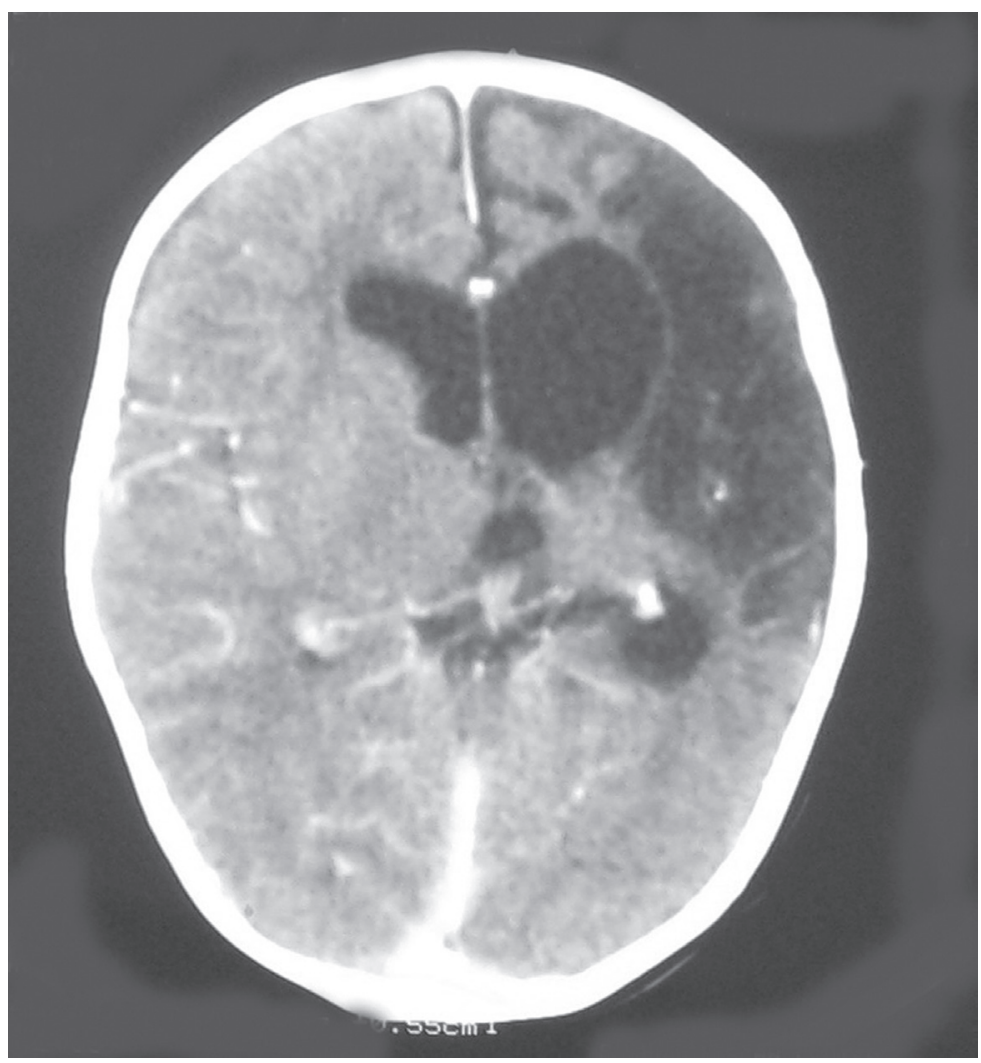

Figure 3 Postoperative brain computed tomography scan 6 weeks after abscess drainage and antibiotic therapy confirms obvious shrinkage of cavities and exvacuum ventriculomegaly.

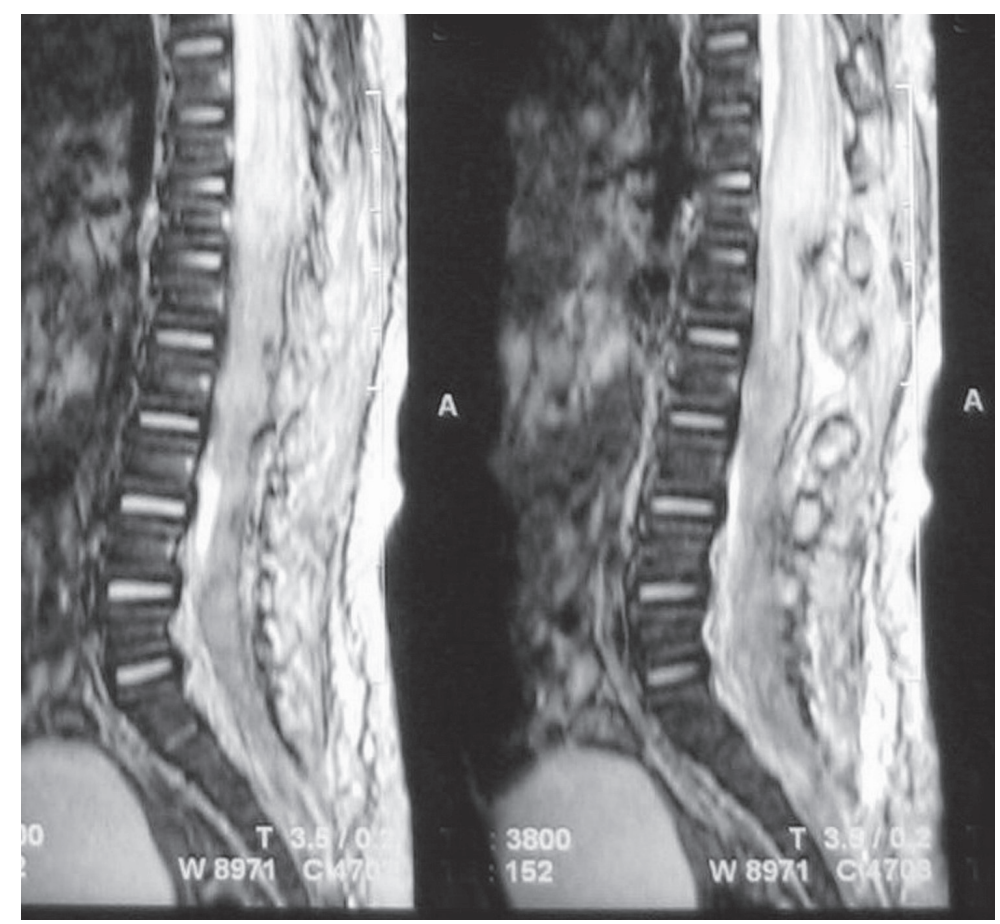

Figure 4 Sagital view,T2-weighted lumbar magnetic resonance image, 2 days after urgent operation is suggestive of cord edema and severe arachnoiditis. 
in the extradural space, and $58 \%$ to $60 \%$ of dermal sinuses terminate in the intradural space (Keating et al 2004). With regard to these termination sites, dermal sinuses have been recognized as potential sites for the entry of bacteria into the intraspinal compartment contributing to the development of recurrent meningitis (Matson and Jerva 1966; Ackerman and Menezes 2003; Keating et al 2004).

As noted previously, brain abscess is a focal infection which is relatively rare especially in infants (Roos and Tyler 2005; Borrego Dominguez et al 2005). Clinical signs of brain abscess are variable which commonly presents as an intracranial mass. Fever is seen in only half of the patients at the time of diagnosis (Roos and Tyler 2005). Presenting manifestations include signs and symptoms of increased intracranial pressure, decreased level of consciousness, seizure and focal neurological deficit (including hemiparesis, aphasia) (Roos and Tyler 2005; Bunyaratavej et al 2006; Carpenter et al 2007).

Known predisposing conditions for brain abscess include: otitis media and mastoiditis, paranasal sinusitis, dental infection, pyogenic lesions in the chest or other body sites, penetrating head trauma, and neurosurgical procedures (Roos and Tyler 2005). Also other underlying causes such as cyanotic heart disease, shunt infection, meningitis, and cranial dermal sinuses have been reported (Ersahin and Mutluer 1994; Brook 1995; Akhaddar et al 2002). In about 5\% of patients the source of infection remains unknown, namely, cryptogenic brain abscess (Moss et al 1988; Ersahin and Mutluer 1994). Spinal abscess is also an uncommon phenomenon. The source of abscess can be hematogenous or contiguous (including dermal sinuses) (Morandi et al 1999; Bunyaratavej et al 2006). Dermal sinus tracts are common predisposing conditions for spinal abscesses especially in children (Moss et al 1988; Bunyaratavej et al 2006).

In our patient, the brain abscess presented with signs of increased intracranial pressure and focal neurological signs (hemiparesis). Efforts made in order to determine the predisposing conditions were all futile. She had not been ill or hospitalized prior to this presentation. There was no previous history of meningitis, contiguous and distant infection, and immune system abnormality. Echocardiography was also normal. Consequently, she was managed as a cryptogenic brain abscess.

On second admission because of spinal abscess, evaluations revealed a dermal sinus tract, harbored at lumbosacral area which was very small to be seen before and purulent discharge was the only clue for discovery. The spinal abscess was the result of direct bacterial extension through the lumbar sinus tract. Occurrence of brain abscess and subsequent spinal abscess after a couple of months in the same child implies more than a mere coincidence. Isolation of the same microorganism from both abscesses supports this assumption. The probable mechanisms of producing intracranial infection in a child with spinal dermal sinus can be meningitis or seeding of pus to subarachnoid space and subsequent cerebritis and abscess. Rarely hematogenous spread of the microorganisms from an infective site inside the spinal canal through bacterial emboli to cerebral tissue may be proposed.

To the best of our knowledge this is the first case of brain abscess associated with a lumbar sinus tract. In this patient, the brain abscess was first considered to be cryptogenic, but lumbar dermal sinus associated with spinal abscess was the probable cause. It can be postulated that a number of brain abscesses that are named cryptogenic, may be due to conditions such as dermal sinuses which are missed and remain undiagnosed.

\section{Conclusion}

Dermal sinus tracts are rather common type of occult spinal dysraphism which commonly manifest during childhood. Although uncommon, it may be considered in a child with a cryptogenic brain abscess. The probable mechanisms of bearing intracranial infection in a child with spinal dermal sinus can be meningitis or seeding of pus to intracranial space and rarely hematogenous spread from an infective site inside the spinal canal related to dermal sinus and then through bacterial emboli to cerebral tissue.

\section{Disclosure}

The authors report no conflicts of interest in this work.

\section{References}

Ackerman LL, Menezes AH. 2003. Spinal congenital dermal sinuses: a 30 year experience. Pediatrics, 112:641-7.

Akhaddar A, Jiddane M, Chakir N, et al. 2002. Cerebellar abscess secondary to occipital dermoid cyst with dermal sinus: case report. Surg Neurol, 58(3-4):266-70.

Ansari S, Dadmehr M, Nejat F. 2006. Possible genetic correlation of an occipital dermal sinus in a mother and son. Case report. J Neurosurg, 105(4 Suppl):326-8.

Bean JR, Walsh JW, Blacker HM. 1979. Cervical dermal sinus and intramedullary spinal cord abscess: case report. Neurosurgery, 5:60-2.

Borrego Domínguez RR, Navarro Gómez M, Gómez-Campderá JA, et al. 2005. Brain abscess in children. Ann Pediatr (Barc), 63:253-8.

Brook I. 1995. Microbiology and management of brain abscess in children. $J$ Child Neurol, 10:283-8.

Bunyaratavej K, Desudchit T, Pongpunlert W. 2006. Holocord intramedullary abscess due to dermal sinus in a 2-month old treated with limited myelotomy and aspiration. J Neurosurg, 104:269-74.

Carpenter J, Stapleton S, Holliman R. 2007. Retrospective analysis of 49 cases of brain abscess and review of the literature. Eur J Clin Microbiol Infect Dis, 26:1-11.

Ersahin Y, Mutluer S, Guzelbag E. 1994. Brain abscess in infants and children. Childs Nerv Syst, 10:185-9. 
Keating RF, Multani J, Cogen P. 2004. Occult spinal dysraphism and the tethered spinal cord. In: Winn HR (ed). Youmans Neurological Surgery, 5th ed. Philadelphia, PA: Saunders, pp. 3257-84.

Matson DD, Jerva MJ. 1966. Recurrent meningitis associated with congenital lumbo-sacral dermal sinus tract. J Neurosurg, 25:288-97.

Morandi X, Mercier P, Fournier HD, et al. 1999. Dermal sinus and intramedullary spinal cord abscess: report of two cases and review of the literature. Childs Nerv Syst, 15:202-6.
Moss SD, McLone, Arditi M, et al. 1988. Pediatric cerebral abscess. Pediatr Neurosci, 14:291-6.

Roos KL, Tyler KL. 2005. Meningitis, encephalitis, brain abscess, and empyema. In: Ksper DL, Braunwald E, Fauci AS, et al. (eds). Harrison's principles of internal medicine, 16th ed. New York: McGraw-Hill, pp. 2471-90. 